\title{
High Global Burden and Costs of Bacterial Vaginosis: A Systematic Review and
} Meta-analysis

\author{
Kathryn Peebles ${ }^{1}$, Jennifer Velloza ${ }^{1}$, Jennifer E. Balkus ${ }^{1,4}$, R. Scott McClelland ${ }^{1,2,3}$, Taunia Markvicka ${ }^{5}$, Joseph L. Amprey ${ }^{5}$, \\ Ruanne V. Barnabas ${ }^{1,2,3,4}$
}

Departments of ${ }^{1}$ Epidemiology, ${ }^{2}$ Global Health, and ${ }^{3}$ Medicine, University of Washington, Seattle, WA; ${ }^{4}$ Vaccine and Infectious Disease Division, Fred Hutchinson Cancer Research Center; ${ }^{5}$ Lupin Ltd.

\section{Background}

Bacterial vaginosis (BV) is the most common vaginal infection among women of reproductive age and is associated with important reproductive and obstetric sequelae, although existing $\mathrm{BV}$ interventions have not demonstrated prevention of most sequelae. Estimates of the burden of BV and associated costs are needed to highlight gaps in epidemiologic evidence and inform research priorities.

\section{Methods}

We conducted a systematic review and meta-analysis of global BV prevalence and costs among reproductive aged women of the general population, excluding, for example, STI clinic attendees and sex workers. We excluded estimates of BV prevalence obtained by methods other than Nugent score. We searched PubMed and Embase, and used random effects models to estimate regional BV prevalence, using separate models for study populations of pregnant women. In cost analyses, we use the most comprehensive published estimate of direct medical costs per BV case for standard treatment in each region. We multiplied these region-specific costs by the annual number of incident cases per region to estimate the costs of treating all cases of BV globally. Assuming a causal relationship, we also estimated the potential costs of BV associated preterm births and HIV cases in the United States.

\section{Results}

Literature search. We identified 2,361 potentially relevant records. After abstract and full-text review, we include data from 122 publications.

Prevalence. Reported BV prevalence is high globally, ranging from 23\% to $29 \%$ in Europe and Central Asia $(23 \%, n=5)$, East Asia and Pacific (24\%, $n=4)$, Latin America and Caribbean (24\%, $n=4)$, Middle East and North Africa $(25 \%, n=2)$, sub-Saharan Africa $(25 \%, n=9)$, North America $(27 \%, n=3)$, and South Asia $(29 \%, n=8)$ (Figure 1). Within North America, African-American and Hispanic women have significantly higher (33\% and 31\%, respectively) prevalence than other racial and ethnic groups (White: 23\%; Asian: 11\%) $(p<0.01)$ (Figure 2).

Economic burden. The cost per treated BV case varied from $\$ 12$ in sub-Saharan Africa to $\$ 90$ in North America (Table 1). The estimated annual global economic burden of treating symptomatic BV cases is $\$ 4.8$ billion (95\% Cl: $\$ 3.7$ billion, $\$ 6.1$ billion) (Table 1$)$. The cost per preterm birth in the US is $\$ 31,400^{4}$ and the annual cost of treatment per person living with HIV is $\$ 25,990^{5}$. When including the direct medical costs of these conditions, the US economic burden of BV is nearly tripled (additional \$3.6 billion), with African-American women bearing a disproportionate burden of these costs.

\begin{tabular}{|lcrrrr|}
\hline \multirow{2}{*}{ Table 1. Global annual economic burden of bacterial vaginosis cases } \\
\hline & $\begin{array}{c}\text { Cost per treated } \\
\text { BV case }\end{array}$ & \multicolumn{2}{c|}{ Snnual cost (95\% confidence interval) (millions 2017 USD) } \\
\cline { 3 - 6 } & & 4,820 & $(3,657,6,130)$ & 13,811 & $(10,479,17,565)$ \\
\hline All regions & $\$ 90^{1}$ & 1,298 & $(1,151,1,449)$ & 3,718 & $(3,298,4,152)$ \\
\hline North America & $\$ 90$ & 1,022 & $(824,1,234)$ & 1,851 & $(1,492,2,235)$ \\
$\quad$ White & $\$ 90$ & 259 & $(223,295)$ & 468 & $(403,535)$ \\
African-American & $\$ 27^{2}$ & 835 & $(670,1,014)$ & 2,391 & $(1,919,2,905)$ \\
Europe \& Central Asia & $\$ 12^{3}$ & 291 & $(247,336)$ & 833 & $(709,963)$ \\
Latin America \& Caribbean & $\$ 12$ & 1,015 & $(717,1,346)$ & 2,909 & $(2,055,3,858)$ \\
East Asia \& Pacific & $\$ 12$ & 914 & $(675,1,172)$ & 2,619 & $(1,935,3,359)$ \\
South Asia & $\$ 12$ & 197 & $(64,371)$ & 564 & $(184,1,064)$ \\
Middle East \& North Africa & $\$ 12$ & 271 & $(132,441)$ & 777 & $(379,1,263)$ \\
Sub-Saharan Africa & & & & \\
\hline
\end{tabular}

Figure 2. Prevalence of BV by race and ethnicity in the United States.

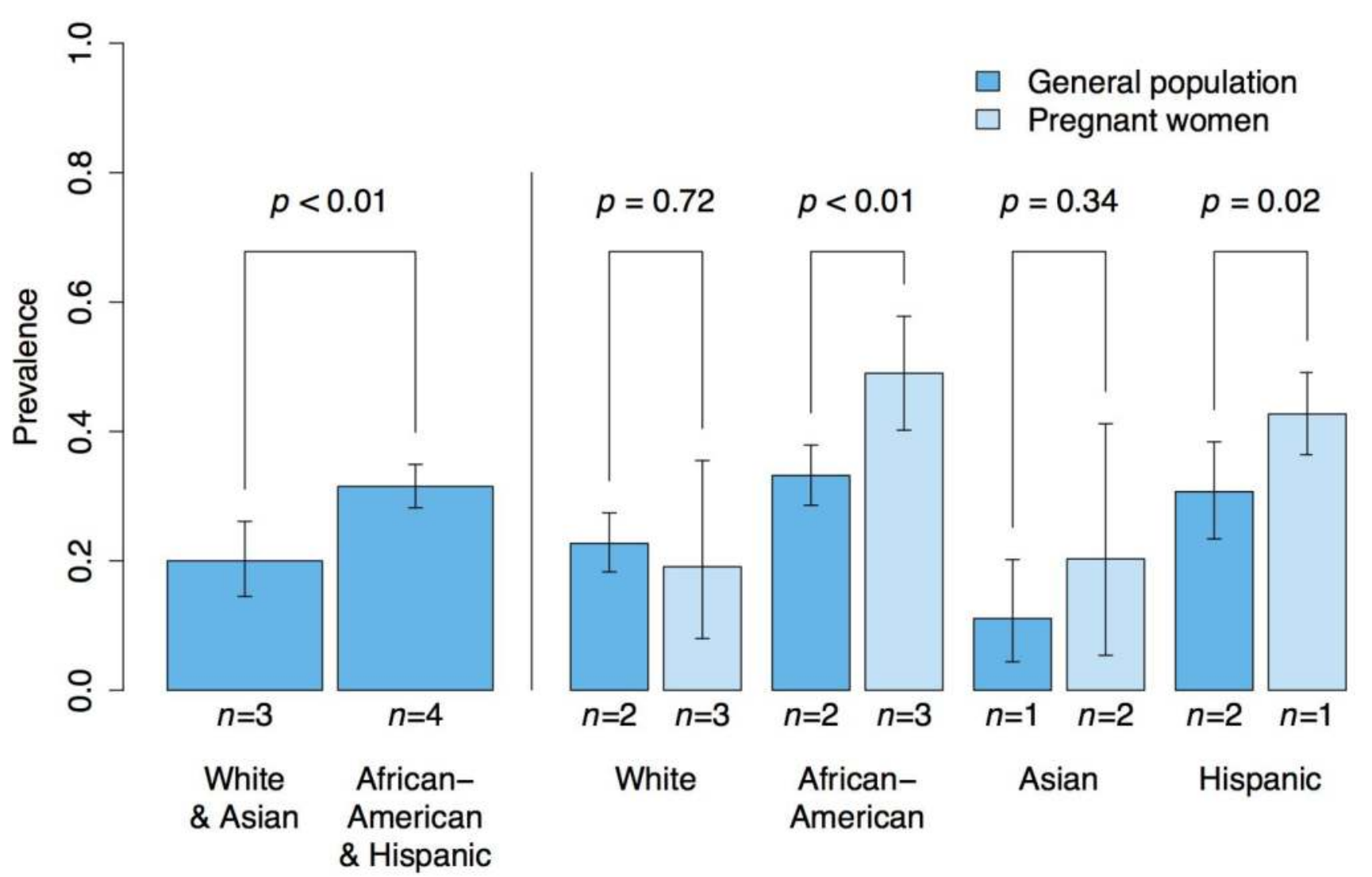

